\title{
Zeitfenster beachten
}

\section{Innovationsbedingte Fortschritte bei Umweltemissionen und -risiken müssen nicht allein Ergebnis gezielter Aktivitäten im Bereich von Umweltinnovationen sein. Sie können sich auch als mehr oder weniger ungeplante Folge einer auf anderweitige Zwecke hin ausgerichteten Innovationstätigkeit einstellen. Vielleicht ließe sich dieser Effekt durch neue Ansätze in der Innovations- und Umweltpolitik verstärkt nutzbar machen.}

$\mathbf{W}_{\text {i }}$

Von Georg Erdmann wird, so denken die meisten an Umweltinnovationen, die unmittelbar auf die Verringerung bestimmter Emissionen und ökologischer Belastungen zielen (1). Manchmal haben derartige Innovationen auch noch weitere Vorteile, aber oft ist dies nicht der Fall, und dann können sie sich am Markt ohne zusätzliche öffentliche Unterstiitzung meist nur schlecht durchsetzen. Dies zeigen beispielsweise etliche Fallstudien im Rahmen des Forschungsverbunds ,Innovative Wirkungen umweltpolitischer Instrumente“. Daneben gibt es aber auch eine Reihe von Innovationen, die nicht gezielt und unmittelbar auf die aktive Verbesserung der Umwelt ausgerichtet sind, sich aber gleichwohl in dieser Weise auswirken. Ein historisches Beispiel ist die Einfiihrung von Elektromotoren in industriellen Produktionsprozessen (2). Vorher erfolgte die Kraftversorgung von einem zentralen Kohlekraftwerk über Wellen und Riemen an die einzelnen Arbeitsplätze, womit die räumliche Organisation der Produktion durch die Geometrie der Energieverteilung festgelegt und sehr inflexibel war. Mit der Einfiuhrung des Elektromotors wurde diese Beschränkung hinfällig und damit rationellere Produktionsstrukturen möglich. Gleichzeitig konnten Umweltvorteile erzielt werden: Mit dem Elektromotor ließ sich die mechanische Energie des Kraftwerks effizienter nutzen (weniger Reibungsverluste). Außerdem wurden durch die Verlagerung des Kraftwerks aus dem Betriebsgelände wesentlich bessere Arbeits- und Wohnumfeldbedingungen möglich (lokale Staubemissionen). Der umweltpolitische Fortschritt darf aber kaum als Ergebnis gezielter Innovationsbemühungen interpretiert werden, sondern ist die ungeplante Folge einer auf andere Zwecke hin ausgerichteten Innovationstätigkeit.
Die innovationsbedingten Umweltvorteile der generellen Innovationstätigkeit müssen natürlich über eine ganzheitliche Betrachtung relativiert werden. In unserem Beispiel (Verlagerung von Kraftwerken an den Stadtrand) wurden die wirtschaftliche Nutzung der Abwärme und damit eine bestimmte Entwicklung hin zu mehr Energieeffizienz (Kraft-Wärme-Kopplung) behindert. Die Basis-Innovation Elektromotor hat also nicht nur zur Lösung vorher bestehender ökologischer Belastungen beigetragen, sondern auch zu einer Verschärfung von Umweltproblemen an anderer Stelle geführt.

Dennoch bleibt die Beobachtung, daß es über die allgemeine Innovationstätigkeit immer wieder zu einer - generellen oder nur punktuellen - Verbesserung der Umweltsituation gekommen ist. Aus Sicht der neoklassischen Ökonomik erscheint dies ziemlich paradox, denn nach dieser Theorie ist der innovationsbasierte Umweltfortschritt durch eine doppelte Externalität behindert: Erstens sind ökologische Schäden in weiten Teilen nicht im Preissystem internalisiert und sollten bei den (Innovations-) Entscheidungen autonomer Wirtschaftsakteure keine Rolle spielen. Zweitens ist der Innovationsprozeß selbst durch Externalitäten gekennzeichnet, denn bekanntlich ist es für den einzelnen innovativen Pionier kaum möglich, den überwiegenden Teil der erarbeiteten Innovationsvorteile für sich zu behalten. Es besteht daher die Neigung, mit möglichst geringen eigenen Innovationsanstrengungen an den Innovationserfolgen anderer Akteure zu partizipieren (free riding). Daraus resultiert die Neigung der Märkte zu sogenannten Innovations-Clustern - also einer Innovationslandschaft, bei der viele Akteure ähnliche Innovationsziele parallel verfolgen und dabei manch andere aussichtsreiche Innovationschance ungenutzt bleibt. Dieser Vorgang bedingt eine zusätzliche Verstärkung des ersten Effekts zu ungunsten eines Umweltfortschritts im Rahmen der allgemeinen Innovatonstätigkeit.

Insgesamt ist es folglich schwer erklärlich, warum aus der allgemeinen Innovationstätigkeit überhaupt positive Auswirkungen auf die Umwelt entstehen. Warum konnte sich beispielsweise die gesamtwirtschaftliche Energieeffizienz (Primärenergieaufkommen pro Einheit Bruttosozialprodukt) in den Industrieländern zwischen 1900 und heute um den Faktor vier verbessern, obwohl die Energiepreise - mit Ausnahme kurzer Zeitspannen - real gesunken sind?

\section{Differenzierung notwendig}

Um dem empirischen Phänomen ungeplanter Umwelffortschritte durch Innovationen näher auf den Grund zu gehen, empfiehlt sich eine Differenzierung des Untersuchúngsgegenstandes in Hinblick auf die folgenden Aspekte:

- Art des betroffenen Umweltbereichs (lokale, regionale oder global wirkende Emissionen, Risiken, Biodiversität, Ressourcenverknappung, Nachhaltigkeit ...),

- Akteurskonstellation auf dem relevanten Markt (Marktgröße und -wachstum, Konzentration auf der Anbieter- resp. Nachfragerseite, Eigentümer, Wettbewerb und Markteintritt, Rolle der staatlichen Nachfrage, Netzwerk-Externalitäten, kulturelle Eigenarten der handelnden Entscheidungsträger ...),

- technisch-ökonomische Innovationsbedingungen und -potentiale (relative Kosten alter und neuer Technologien inkl. Entwicklungsperspektiven, sunk costs, Innovationsstadien der relevanten Innovationsaktivitäten, Investitionszyklen ...).

Den Einsichten der ökonomischen Innovationstheorie zufolge muß ein Einfluß der genannten ökologischen, wirtschaftlichen und technischen Strukturmerkmale auf die Innovationstätigkeit

\section{Beilagenhinweis}

\section{Unsere Publikation enthält ein Konferenzhinweis und Programm von IÖW und VÖW}

Wir bitten um freundliche Beachtung. 
und den Innovationserfolg unterstellt werden. Beispielsweise dürften Innovationsaktivitäten Ressourcenverknappungen und Risiken eher berïcksichtigen als globale Emissionen oder Biodiversität, denn die erstgenannten Gesichtspunkte könnten sich - anders als die letztgenannten - als ein Hindernis für die Durchsetzung der angestrebten Innovation am Markt erweisen. In ähnlicher Weise dürften Innovationen eher mit ökologischen Vorteilen verbunden sein, wenn sie nicht dem Zeitdruck eines Innovationswettlaufs (time to market) unterliegen, weil dann eine sorgfältige Technikfolgenabschätzung möglich ist.

\section{- Ein Beispiel für Fehlsteuerung}

Ein besonderes Augenmerk verdient die Betrachtung der zeitlichen Dynamik von technisch-ökonomischen Innovationsbedingungen. Daß sich bei einer Analyse gerade dieses Gesichtspunkts schwerwiegende politische Fehlsteuerungen der Innovationstätigkeit vermeiden ließen, läßt sich an vielen Beispielen belegen. Eines davon ist die Technik der Chlor-AlkaliElektrolyse, bei der es um die Gewinnung von Chlor, Wasserstoff und Natronlauge geht. Heute gibt es drei marktgängige Verfahren:

das Amalgam-Verfahren, welches mit dem ökologischen Nachteil von Quecksilber-Emissionen verbunden ist,

- das Diaphragma-Verfahren, welches AsbestEmissionen verursacht, und

das erst seit Ende der 70er Jahre kommerziell verfügbare Membran-Verfabren, welches ökologisch (vergleichsweise) unbedenklich ist und einen geringeren Energieverbrauch aufweist - vor allem aber verfahrenstechnisch einfacher und damit deutlich kostengünstiger ist.

\section{Weitere Literatur zum Schwerpunkt}

Zu sozialen und institutionellen Innovationen vgl. insbesondere

- Horst Zimmermann, Nils Otter, Dieter Stahl, Matthias Wohltmann: Innovation jenseits des

Marktes. Neverungsverhalten in Staat, privaten Haushalten und Nonprofit Organisationen und der Einfluß umweltpolitischer Instrumente. Analytica, Berlin 1998.

Für die internationale Diskussion vgl. insbesondere - Rene Kemp: Environmental Policy and Technical Change. A Comparison of the Technological Impact of Policy Instruments. Edward Elgar, Cheltenham 1997.

und die jeweils dort angegebene literatur.
Trotz der wirtschaftlichen Vorteile hat das Membran-Verfahren einen Weltmarktanteil von (1991) erst 20 Prozent. In Deutschland dominiert zur Zeit das Amalgam-Verfahren mit einem Marktanteil von über 60 Prozent, das MembranVerfahren spielt (noch) keine Rolle. Ähnlich sieht es in Westeuropa aus (3).

Nach Betge erklärt sich dieser paradoxe Zustand dadurch, daß der Investiti onszyklus in dieser Branche gegen Ende der 70er Jahre durch den damals verschärften gesetzlichen Emissionsschutz unterbrochen wurde: Mit beträchtlichem Investitionsaufwand wurden bestehende Amalgam- und Diaphragma-Anlagen ökologisch nachgerïstet - und dies zu einem Zeitpunkt, als das innovative Membran-Verfahren kurz vor der Markteinführung stand (4). Die damals erzwungenen antizyklischen Investitionen lasten noch heute auf dem Markt, der inzwischen durch Überkapazitäten gekennzeichnet ist (5).

\section{Vorfeil flexibler Regulierung}

Einen anderen Ansatz verfolgte die Innovationspolitik in Japan: Der Gesetzgeber bzw. Regulierer erzwang Ersatz-Investitionen erst zu einem Zeitpunkt, als die Altanlagen im Rahmen des regulären Investitionszyklus ersetzt werden mußten und die neue Membran-Technologie kommerziell vorteilhaft geworden war. Diese hat heute in Japan einen Marktanteil von über 70 Prozent. Ökologie und Ökonomie können damit von einer Zeitstrategie der umweltpolitischen Regulierung profitieren, bei der ein ursprünglich ökologisch unbefriedigender Zustand kurzfristig hingenommen und eine längerfristig vorteilhafte innovative Lösung abgewartet wurde.

Diese Kunst eines innovationsorientierten, zeitlich flexiblen Handelns läßt sich auch außerhalb Japans beobachten. Ein interessantes Beispiel sind die Umweltschutz-Leitlinien für die US-amerikanische Papierindustrie, mit denen offenbar erstmalig eine Flexibilisierung von Umweltschutz-Auflagen erfolgt (6): Die Unternehmen der Papierindustrie können sich für eine von drei Optionen (tiers) entscheiden, die sich durch die zu erreichenden Grenzwerte, durch unterschiedlich lange Anpassungszeiträume (bis zu 15 Jahren!) und durch differenzierte administrative Erleichterungen (Meß- und Verwaltungsaufwand, öffentliche Kontrollen etc.) unterscheiden. Überträgt man die Erfahrungen aus dem Beispiel Chlor-Alkali-Elektrolyse, darf vermutet werden, daß der reguläre Innovations- prozeß in der amerikanischen Papierindustrie ökologische Fortschritte erlauben wird, ohne daß dies mit einer zusätzlichen Kostenbelastung verbunden sein wird.

In Europa hingegen betrachten viele Ökonomen und Ökologen flexible, ,weiche“ Ansätze der Umweltpolitik als den Versuch von Interessengruppen, sich um wirksame ökologische Fortschritte drücken zu können (7). Das Schlagwort des No-Regret-Potentials kann diese intellektuelle Denkfalle nicht schöner formulieren: Ein wirklicher ökologischer Fortschritt wäre demnach nur möglich, wenn mehr als nur die vorhandenen wirtschaftlich attraktiven Maßnahmen zur Umweltentlastung ergriffen werden. Kaum jemand stellt sich der Frage, wie dies No-RegretPotential durch zielgerichtetes, aber zeitflexibles Einwirken auf den generellen Innovationsprozeß vergrößert werden könnte. Hier liegen Forschungsfragen brach, die einer dringenden Bearbeitung harren.

\section{Anmerkungen}

(1) Hemmelskamp, J.: Umweltpolitik und Innovation Grundlegende Begriffe und Zusammenhänge. Zeitschrift für Umweltpolitik und Umweltrecht 4/1997, S. 481-511.

(2) Devine, W.D. Jr.: From Shafts to Wires: Historical Perspectives on Electrification. Journal of Economic History 43 (1983), S. 347-372.

(3) Vgl. Onken, U./ A. Behr: Chemische Prozeßkunde. Lehrbuch der technischen Chemie Bd 3. Stuttgart, New York 1996, S. 497 und

Gilliatt, B.S./ H. Schubert: Die Chlorproduktion nimmt weltweit noch zu. Chemische Rundschau 6 vom 21. März 1997

(4) Vgl. Betge, T.: Energieeffizienz in der Industrie: Marktversagen bei der Chlor-Alkali-Elektrolyse. Diplomarbeit. TU Berlin 1998, S. 49ff.

(5) Vgl. Staab, R./ D. Bergner/ W. Scheibnitz: Umrüstung auf Membranzellen in der Alkalichlorid-Elektrolyse vor dem Hintergrund der FCKW/CKW-Diskussion. Chemie Ingenieur Technik 65 (1993), S. 1337-1340.

(6) Blazejczak, J./ D. Edler: Innovative Ansätze zur Förderung von Umweltinnovationen in der Papierindustrie in den USA und Schweden. Manuskript eines Forschungsberichts. Deutsches Institut für Wirtschaftsforschung, Berlin 1997. (7) Vgl. z.B. European Environmental Agency (ed.): Environmental Agreements: Environmental Effectiveness. Vol I and II. Kopenhagen 1997.

\section{Der Autor}

Dr. Georg Erdmann ist Professor an der TU Berlin. Kontakt: Fachgebief Energiesysteme der TU Berlin, Einsteinufer 25, 10587 Berlin, E-mail: erdmann@ensysi.fb10.tu-berlin.de 
(c) 20I0 Authors; licensee IÖW and oekom verlag. This is an article distributed under the terms of the Creative Commons Attribution Non-Commercial No Derivates License (http://creativecommons.org/licenses/by-nc-nd/3.o/), which permits unrestricted use, distribution, and reproduction in any medium, provided the original work is properly cited. 